\title{
Factores divergentes entre las economías ecológica y ambiental: el caso Conga
}

\author{
Econ. Christiam Méndez Lazarte \\ Universidad de Lima
}

Recibido: 09/07/2015 / Aprobado: 03/12/2015

\begin{abstract}
Resumen: Este artículo contrasta las diversas opiniones de personas y/o instituciones involucradas en el caso Conga, identificando aspectos comunes y divergentes entre las organizaciones, así como de los líderes, en cada uno de los temas que propone el marco teórico. El análisis de la información obtenida sugiere que los conflictos ambientales se sustentan en las diferencias entre los enfoques económicos que asumen los líderes de las comunidades campesinas y los representantes del Estado, puesto que los primeros tienen un enfoque basado en la economía ecológica, mientras que las leyes del Estado y sus representantes no establecen con claridad una posición entre la economía ecológica y la ambiental. Esta divergencia no solo se da en el Perú, sino también en otros países de América Latina, lo que debe motivar un debate que cuestione los fundamentos de la política ambiental en la región.
\end{abstract}

Palabras clave: economía ecológica / economía ambiental / conflictos socioambientales / minería / Conga

\section{Divergent Factors Between Ecological's Economy and Environmental's Economy: Conga Case}

АвsтRAст: This article compares the different views of individuals and/or organizations involved in Conga case, identifying common and divergent aspects between organizations and leaders in each of the issues proposed in the theoretical framework. The analysis of information obtained suggests that environmental conflicts are based on differences between the economic approaches assumed by peasant community leaders and the Government representatives, since the first supports an ecological-economic approach, while the Government Law and its representatives do not establish a clear position between Ecological and Environmental Economics. This divergence occurs not only in Peru but in other Latin American countries, which should encourage a debate about the foundations of regional environmental policy.

Keywords: ecological economics / environmental economics / socio-environmental conflicts / mining / Conga 


\section{INTRODUCCIÓN}

Esta investigación se justifica no solo en el impacto que tienen en el Perú los conflictos socioambientales, sino también en América Latina, como ya lo vienen manifestando algunos autores como Tábara (2003) y Walter (2008).

Los proyectos extractivos han sido la base de la expansión de los países de América Latina en los últimos 10 años. Esta clase de proyectos han coincidido con una tendencia mundial y local hacia la conservación ambiental y el empoderamiento de las comunidades que conviven con los proyectos mineros. Por tal razón, es importante encontrar mecanismos que permitan reducir los conflictos socioambientales y hallar puntos de consenso entre las empresas extractivas, el Estado y las comunidades aledañas, pero también entre las instituciones que conforman el tejido social, como las universidades, organizaciones no gubernamentales o instituciones internacionales, que con sus opiniones influyen en los conflictos.

Dos investigadores de la minería peruana como Glave y Kuramoto plantean la necesidad de seguir estudiando los conflictos sociales relacionados con la actividad extractiva (Glave y Kuramoto, 2007). Las actividades mineras son la principal fuente de conflictos entre la empresa, la comunidad y el Estado peruano. De los casos en prevención, el $49 \%$ están relacionados con el sector minero, y de los casos activos, 16 de 43 son mineros; estas cifras la catapultan en el sector con la más alta incidencia. Finalmente, de los 16 casos, 5 se han generado en Cajamarca, siendo el departamento que más conflictos concentra.

Cuadro 1

Casos de prevención de conflictos según tipo

\begin{tabular}{lrr}
\hline \multicolumn{1}{c}{ Tipo } & Número & $\%$ \\
\hline Minería & 51 & 49 \\
Hidrocarburos & 11 & 10 \\
Minería informal & 1 & 1 \\
Agrarios & 3 & 3 \\
Laborales & 2 & 2 \\
Demarcación territorial & 3 & 3 \\
Hídricos & 6 & 6 \\
Energéticos & 8 & 8 \\
Otros & 20 & 19 \\
Total & 105 & 100 \\
\hline
\end{tabular}

Fuente: Sistema de Información de la Oficina Nacional de Diálogo y Sostenibilidad (ONDS), actualización al 30 de setiembre de 2014 
El enfoque de la economía ecológica aporta una estructura más sólida a los argumentos orientados a que no se realicen las actividades mineras. Esta investigación nos ayuda a explicar la posición que están tomando algunos actores o líderes de opinión con respecto a la ejecución de proyectos extractivos con impactos negativos en el ambiente (Martinez Allier, 2004), quienes a su vez proponen una orientación hacía la conservación (Naredo, 2011).

Por otro lado, la economía ambiental planteada se orienta en la teoría clásica de la economía de las fallas en el mercado y toma el concepto de externalidad (García, Kristrom, y Brannlund, 2005). A diferencia de la economía ecológica, criticada duramente por la economía clásica, la economía ambiental se sirve de ella y plantea una mejora (Xercavins Valls, Sabater Pruna, Cervantes Torre-Marín, y Cayuela Marín, 2005).

Producto de la revisión bibliográfica, se han identificado cuatro ejes de debate que marcan los dos enfoques económicos, los cuales hemos denominado componentes divergentes.

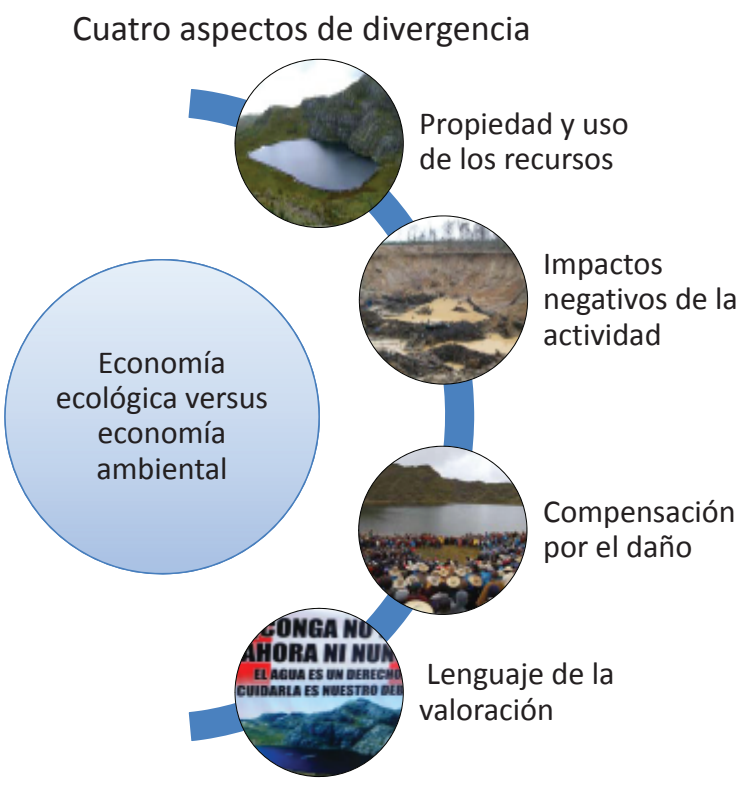

Figura 1: Cuatro componentes divergentes entre la economía ecológica y la economía ambiental

Fuente: Elaboración propia (imágenes obtenidas de páginas webs) 
Desde la perspectiva de la economía ecológica, la propiedad es el tema principal de debate (Aguilera Klink y Alcántara, 1994), y por tanto la opinión de las comunidades aledañas sobre el uso y administración del bien público debe ser vinculante. Esto le da un sentido de propiedad a las comunidades y gobiernos locales de zonas aledañas (Walter, 2008), que forma parte del origen de los conflictos ambientales en la discusión sobre la propiedad, como plantea Martinez (Martinez-Alier, 2012).

En una visión clásica de la economía, el conflicto se origina por la escasez de los recursos (García, Kristrom, y Brannlund, 2005). Por su parte, el mayor representante de la economía ambiental, Ronald Coase, menciona que los problemas ambientales se generan debido a la seguridad legal de la propiedad privada. Por tanto, los recursos deben tener un propietario y el Estado debe garantizar la normatividad (Coase, 1960).

Otro componente de debate entre los actores fue la apreciación de los impactos que genera una actividad económica sobre los ecosistemas (De Echave, Diez, Huber, Revesz, Ricard, y Tanaka, 2009).

En este caso, la economía ambiental considera que los impactos se pueden mitigar o neutralizar, y que son clasificables (Mendezcarlo Silva, Medina Jiménez, y Becerra Quintero, 2010). Mientras que para la economía ecológica, la segunda ley de la entropía es uno de los sustentos relacionados a la irreversibilidad que ocasionan las actividades económicas y que estas, al transformar la materia, generan un excedente que no puede ser reciclado ni recompuesto (Georgescu-Roegen, 1970).

La posición de la economía ecológica ligada a la biología menciona la importancia de la participación de las generaciones futuras y la vulnerabilidad de los pobres (Martinez Allier, 2010).

Otro componente divergente es la compensación por el daño. En ese aspecto, la economía ambiental propone la monetarización y el pago de un impuesto como reparación (García, Kristrom, y Brannlund, 2005) (Mendezcarlo Silva, Medina Jiménez, y Becerra Quintero, 2010). Pero la economía ecológica no piensa igual. Por ejemplo, Kapp consideró que las actividades económicas generan costos sociales y que afectan a otras personas o a la sociedad (Kapp, 1950), aunque, debido a que los empresarios no se consideran responsables, ello produce que este se apropie de una parte de la producción nacional que no le corresponde.

Frente a la percepción de un impacto irreversible en el ecosistema y de una magnitud que trasciende en el tiempo a los seres humanos que en ese momento pueden tomar una decisión, la compensación monetaria no es aceptada por el enfoque de la economía ecológica (Martinez-Alier, 2012). 
El valor económico de un recurso natural se diferencia entre los valores de uso y no uso desde la perspectiva de la economía ambiental, así como de los valores actuales y futuros (Luke Brander, 2010). Sin embargo, para Martinez Alier: "Hay una asimetría en la forma de tratar la depreciación del capital y el desgaste o pérdida de recursos naturales" (Martinez Alier, 1998) y que la valoración de un recurso natural sea inconmensurable está en la tradición de la economía ecológica desde William Kapp (Kapp, 1970).

Finalmente, dentro de la economía ecológica también está la posición relacionada a la biología, donde se propone:

Sobre la conexión entre termodinámica y economía convencional, y si en todos los procesos físicos, cualquiera que sea su naturaleza se pierde energía utilizable, es razonable pensar que la función del coste energético pueda ser una base objetiva para medir los costes físicos de producción. Nótese -precisábamos entonces- que no estamos diciendo su precio o su valor sino los costes medidos en unidades de recursos empleados (Naredo, 2011).

La proposición principal de esta investigación es que los conflictos socioambientales se originan por las diferencias de opinión que existen entre los actores involucrados con el caso Conga, asumiendo, de manera consciente o no, una posición vinculada con la economía ecológica o ambiental. Cabe señalar que no existe una línea de investigación empírica que proponga esta afirmación. Esto se puede deber al proceso de evolución y difusión de la economía ecológica y ambiental en América Latina.

El caso Conga se da en un contexto donde la minería y los hidrocarburos comprenden un gran porcentaje de las exportaciones en el Perú, y a que en los últimos años ha existido un comportamiento altamente favorable de los precios de los productos mineros, lo que ha promovido la dependencia del crecimiento económico del Perú con esta actividad (Centro Nacional de Planeamiento Estratégico, 2010). En ese sentido, es importante tomar en cuenta que la minería es la cuarta actividad extractiva y representa el $5,1 \%$ del PBI, alrededor del 40 a 50 por ciento de las exportaciones del país, lo que genera empleo directo a 211748 personas en 22 regiones.

Durante años los planteamientos políticos han estado orientados a que las empresas mineras transformen el insumo incrementando su valor agregado, pero esto ha tenido muy bajos resultados, por la alta conveniencia económica que resulta de su exportación.

El proyecto Conga es aurífero y requiere de una inversión de US $\$ 4800$ millones de dólares, lo que brindaría un canon para Cajamarca de 150 millones de soles y la posibilidad de abrir un fondo social por 130 millones 
de soles. Como parte del proyecto y en compensación por los daños se ha propuesto la creación de cuatro reservorios artificiales de agua (Perol, Chailhuagón, Superior e Inferior) con 13 millones de metros cúbicos de agua prometidos. Este proyecto espera encontrar, además de oro, diez metales pesados entre: aluminio, zinc, cadmio, hierro y plomo.

Los riesgos ambientales estimados son contaminación del agua de superficie y subterráneas o su agotamiento, contaminación del aire, inseguridad alimentaria (daños a los cultivos), calentamiento global, pérdida del paisaje, deforestación y reducción de cobertura vegetal, contaminación del suelo y erosión del suelo.

Mientras que los riesgos sociales son: desplazamiento de la población, aumento de la violencia y delincuencia, despidos y desempleo, pérdida de conocimientos tradicionales y aumento de la corrupción. Adicionalmente, se considera que los campesinos son susceptibles de perder la tierra y extremadamente vulnerables a los riesgos ambientales y financieros, lo que genera enfrentamientos entre la población y las autoridades.

El caso Conga es emblemático y por ese motivo ha sido sensible de comentarios y posiciones de diferentes instituciones y líderes a nivel nacional, lo que invita a profundizar en los orígenes del conflicto y sus argumentos desde una perspectiva económica.

\section{METODOLOGÍA}

La investigación es cualitativa, de carácter exploratorio y basada en la metodología de casos. Tomando como referencia a Robert Yin (Yin, 1984) y Marthew y Humberman (Matthew, 1994), se ha considerado como unidad de análisis la opinión de las personas y/o instituciones involucradas en el caso Conga. Estas opiniones son el elemento central de la investigación, puesto que manifiestan los argumentos frente a diversos temas vinculados con el conflicto. Las opiniones seleccionadas pudieron ser vertidas tanto en el momento del conflicto como posteriormente e incluso en la actualidad, al igual que a propósito de otros conflictos socioambientales o del tema en general, tomando como referencia los componentes divergentes previamente determinados.

El muestreo convino dos técnicas no probabilistas, considerando la naturaleza cualitativa de la investigación. El primero fue un muestreo por conveniencia y el segundo un muestreo por snowball (Malhorta, 2008).

En la primera técnica de muestreo se mapeó a los principales actores, así como a las personas que representaron en ese momento la posición de 
cada uno de los actores que participaron directa o indirectamente del conflicto. La selección de estos actores y sus representantes ha sido producto de un análisis exploratorio del caso Conga, tomando a las instituciones relacionadas con los documentos generados. Posteriormente, se aplicó la técnica de "bola de nieve", donde los entrevistados, documentos, declaraciones en medios nos sugieren instituciones y personas relevantes que pueden ser contactadas.

Se aplicaron múltiples fuentes para obtener evidencia de las opiniones: documentación, entrevistas a profundidad y reunión focalizada, y declaraciones a los medios, tomando como referencia a Robert Yin, quien es regularmente citado por investigadores científicos (Yin, 1984).

Para la aplicación de las entrevistas a profundidad se diseñó una guía de indagación para conocer la posición de las personas frente el conflicto de Conga y los argumentos que sustentan su opinión, para luego contrastarlo con la posición de la institución (Malhorta, 2008).

La cantidad de entrevistas ha sido determinada por múltiples factores como el punto de saturación y la disponibilidad de los entrevistados de ser contactados. Se tomó como referencia una investigación realizada a expertos por Baker y Edwards como parte de una publicación del National Center for Research Methods Review Paper, donde mencionan: “La cantidad de casos en investigación cualitativa no tiene carácter determinista a diferencia de la investigación cuantitativa y está condicionada a la peculiaridad de cada proyecto y sobre la base del criterio del investigador" (Baker y Edwards, 2012).

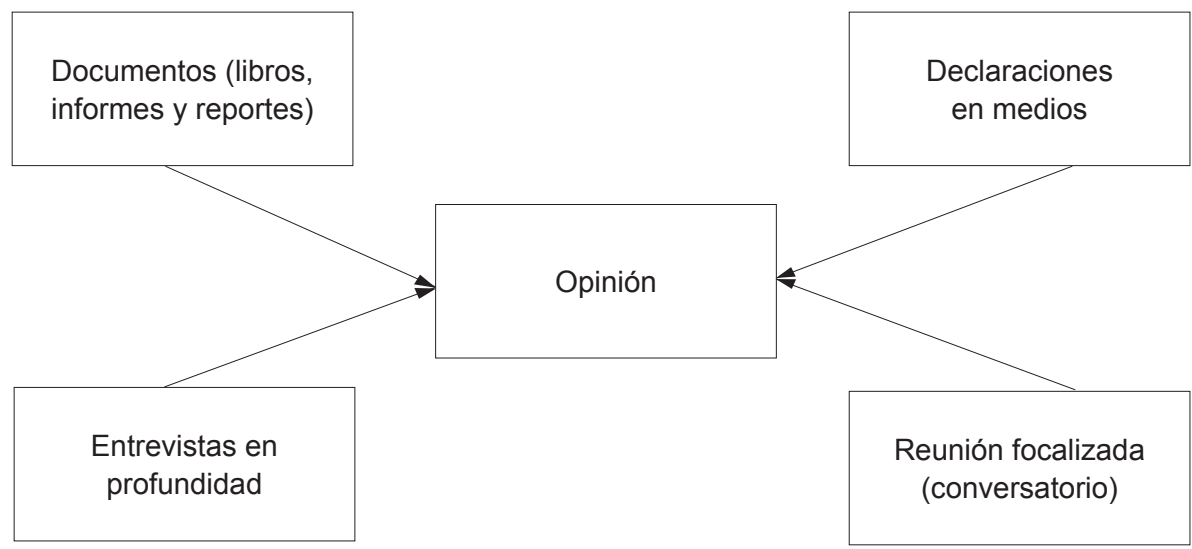

Figura 2: Convergencia de múltiples fuentes de evidencia Fuente: Elaboración propia tomando como referencia a Roberto Yin en Case Study Research (Yin, 1984) 
Finalmente, se realizó una reunión focalizada, convocada como un conversatorio sobre conflictos socioambientales con personas representativas del caso Conga y especialistas, lo que permitió validar los resultados y ajustarlos para identificar conclusiones que podrían reducir la posibilidad de mayores conflictos entre empresas mineras y comunidades campesinas.

Las opiniones y sus argumentos han sido analizados tomando como referencia a Matthew y Huberman en Qualitative Data Analysis: an expanded sourcebook, donde mencionan matrices, flujos, mapas cognitivos y clusters como herramientas analíticas, aplicadas como parte de esta investigación (Matthew, 1994).

Algunas de las herramientas creadas para el análisis de las opiniones y sus argumentos han sido:

- Matriz de componentes divergentes entre la economía ecológica y la ambiental.

- Matriz de componentes divergentes por actores.

- Contraste entre los flujos de opiniones con respecto a la propiedad, impacto negativo de la actividad, compensación y valoración.

- Mapa cognitivo: perspectiva de las causas del conflicto socioambiental en Conga.

- Matriz de inclusión de los componentes de divergencia en el estudio de impacto ambiental.

- Mapa cognitivo: convergencia de opiniones de los actores en los componentes divergentes.

- Mapa cognitivo de los entrevistados.

Esto ha permitido la identificación de los aspectos comunes y divergentes entre las instituciones, así como de los líderes, en cada uno de los temas que propone el marco teórico, acompañados por citas y verbalizaciones obtenidas de diversas fuentes.

\section{RESULTADOS}

La investigación ha logrado identificar cuatro aspectos, en donde se generan diferencias entre los enfoques de la economía ecológica y la economía ambiental, denominados por esta investigación como componentes divergentes: propiedad, medición de los impactos negativos, compensación por el daño y valoración.

La investigación sostiene que los conflictos socioambientales están orientados por las diferencias de opiniones que existen entre los actores 
involucrados en el caso Conga con respecto a los componentes divergentes: propiedad, uso y administración de los recursos, la medición de impactos negativos, la compensación por el daño y en menor medida la valoración.

El recurso agua y su administración han sido la principal fuente del conflicto mediático (De Echave y Diez, 2013). Los argumentos de defensa del agua se relacionan con un sentido de pertenencia de los recursos ambientales, como lo propone Edy Benavides, presidente del Frente de los Intereses, Derechos y Ambiente de Hualgayoc-Bambamarca (La República, 2013). Así también lo manifestaron en letreros y pancartas, como lo evidencia una foto publicada en un medio digital (Quispe, 2012).

Emilio Santos, historiador ecológico, señala que la propiedad es uno de los componentes divergentes que más enfrentamientos puede generar entre la población, la empresa y el Estado (Méndez Lazarte, 2015), como se puede leer en la nota de prensa publicada por el diario Perú 21 (Perú 21, 2013), donde se mencionan los enfrentamientos entre los pobladores y el Estado por este tema.

El debate sobre si el agua es un bien de libre acceso o un bien público, para luego convertirse en un bien privado, es uno de los argumentos del debate, según propone un representante del Estado (ex Ministro del Ambiente y opositor al proyecto Conga, Ricardo Giesecke, Sara Lafosse), puesto que las lagunas que eran un bien de libre acceso, con el proyecto y la compensación (los reservorios de agua) pasarían a ser un bien privado (Méndez Lazarte, 2015).

El reclamo por la propiedad y/o las restricciones de su uso, no solo están orientadas al derecho de las poblaciones aledañas al proyecto, sino también a la capacidad de la municipalidad de restringir su libre uso, como lo mencionó Marco Antonio Arana Zegarra, fundador y militante del partido político Tierra y Libertad (Maquet, 2011).

Como indicó un asesor del Ministerio del Ambiente (Minam) en el conversatorio sobre conflictos socioambientales, existe la necesidad de un cambio de enfoque de la política pública (Méndez Lazarte, 2015).

El representante de una ONG opositora al proyecto propone que "el agua es un elemento articulador que trasciende los límites de las áreas de influencia" y que existe la necesidad de una mirada de mayor amplitud. Además, considera que Yanacocha sería la que moviliza y controla los reservorios, y por tanto desconoce la capacidad del gobierno central de gestionar el recurso (Méndez Lazarte, 2015). 
La posición del gobierno estuvo condicionada a las personas que tuvieron los cargos públicos, lo que denota un debate de posiciones incluso desde su interior. El ex ministro Giesecke elaboró durante su gestión un informe donde se indica que dos lagunas serían utilizadas como botaderos de desechos contaminados durante el proceso minero (Ele, 2012).

Finalmente, como uno de los últimos acontecimientos, en diciembre de 2014 la Sala Penal de Apelaciones de Cajamarca le dio la razón a Máxima Acuña con respecto a una demanda interpuesta por la minera Yanacocha, con el propósito de retirarla de lo que ella considera su propiedad, ubicada frente a la Laguna Azul, una de las áreas que serían afectadas por el proyecto Conga (La República, 2015).

Los hechos y las opiniones de los actores que se oponían al proyecto Conga han estado relacionados con un reclamo sobre la propiedad, uso y administración de los recursos naturales, como lo mencionan las posiciones vinculadas a la economía ecológica. No se llegó a plantear el argumento de que la propiedad le corresponde a las generaciones futuras, aunque este se podría encontrar en la misma línea que la defensa plantea, valiéndose del argumento que es de ellos y de sus hijos.

Lo propuesto por la ley es claro, como lo menciona una funcionaria del Ministerio del Ambiente, pero la población tiene un reclamo que cuestiona la ley (Ministerio del Ambiente, Funcionaria del Ministerio del Ambiente, 2014).

Cabe mencionar que para el representante de Yanacocha, el tema propiedad no fue considerado como primordial, aspecto en el que coincidieron el representante de una ONG y del Ministerio del Ambiente, mientras que para un representante de una ONG opositora al proyecto, el conflicto sí fue un tema de propiedad, puesto que se dudaba de quién administraba el recurso agua (Méndez Lazarte, 2015).

Otro componente divergente fue la veracidad del estudio de impacto ambiental, lo que ha generado que múltiples instituciones no solo cuestionen la solidez técnica del mismo, sino la capacidad de las instituciones públicas para evaluar dichos estudios.

Para los representantes del Minam, los Estudios de Impacto Ambiental (EIA) son documentos muy completos y prevén todos los temas necesarios (Ministerio del Ambiente, 2014), mientras que para Roberto Parra, actualmente Gerente de Proyecto en Indiseo y ex Ingeniero de Proyecto en Knight Piésold, empresa responsable del estudio de impacto ambiental de Conga, "los EIA en la raíz están mal porque son aprobados por una 
autoridad que tiene conflictos de intereses; es la misma que promueve la inversión. Deberían estar en el Ministerio del Ambiente". Además indica: "Como lo ha dicho el Banco Mundial y la Defensoría del Pueblo, los límites máximos permisibles y los estándares de calidad ambiental que tenemos para el sector minero son desactualizados: tienen más de 28 años de antigüedad. Es decir, los EIA no garantizan que no habrá problemas de escasez, ni de contaminación de agua" (Ponce, 2011). En ese mismo sentido, para un ex representante del Ministerio del Ambiente, la contratación de la consultora es una relación entre privados y el contacto es permanente entre ambos, lo que puede poner en cuestionamiento la objetividad de la evaluación (Méndez Lazarte, 2015).

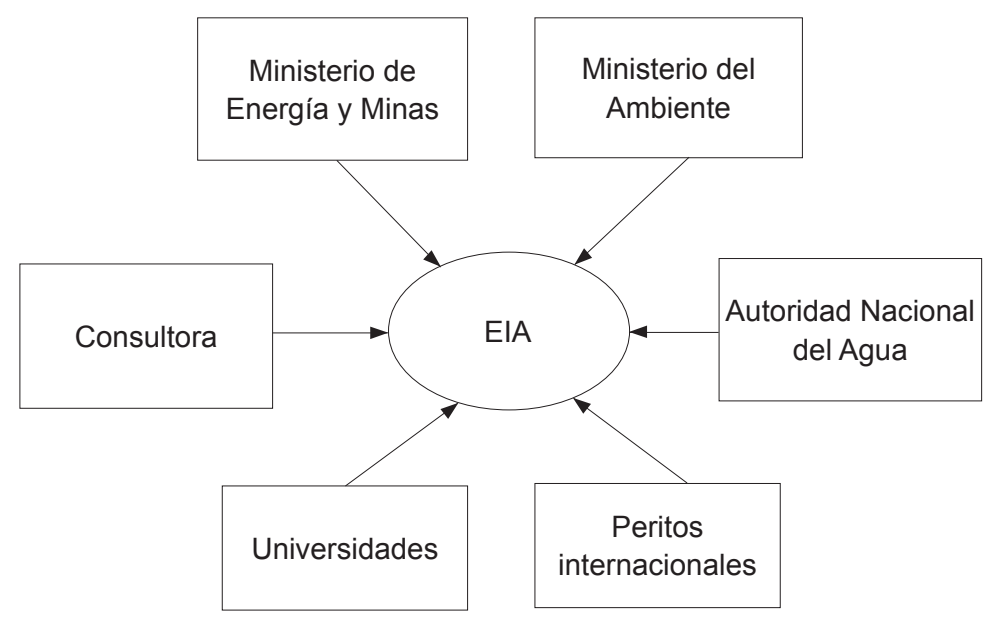

Figura 3: Instituciones que revisaron el Estudio de Impacto Ambiental Fuente: Elaboración propia

Este debate generó que Robert E. Morán, un investigador americano, realizara comentarios a solicitud del Enviromental Defender Law Center. Sus argumentos más importantes se podrían resumir en los siguientes puntos (Morán, 2012):

- Existen conflictos de interés de los evaluadores, puesto que son contratados y pagados por la empresa.

- Cuestiona la calidad del informe por su subjetividad en la redacción o exposición de motivos.

- Pone en cuestionamiento las fuentes de información para medir los impactos y menciona que este no sería aprobado en países desarrollados. 
Por otro lado, el Estado peruano contrató un peritaje internacional orientando a las afectaciones sobre el agua, donde los resultados son favorables para la realización del proyecto. Se plantea la creación de reservorios como compensación por los daños.

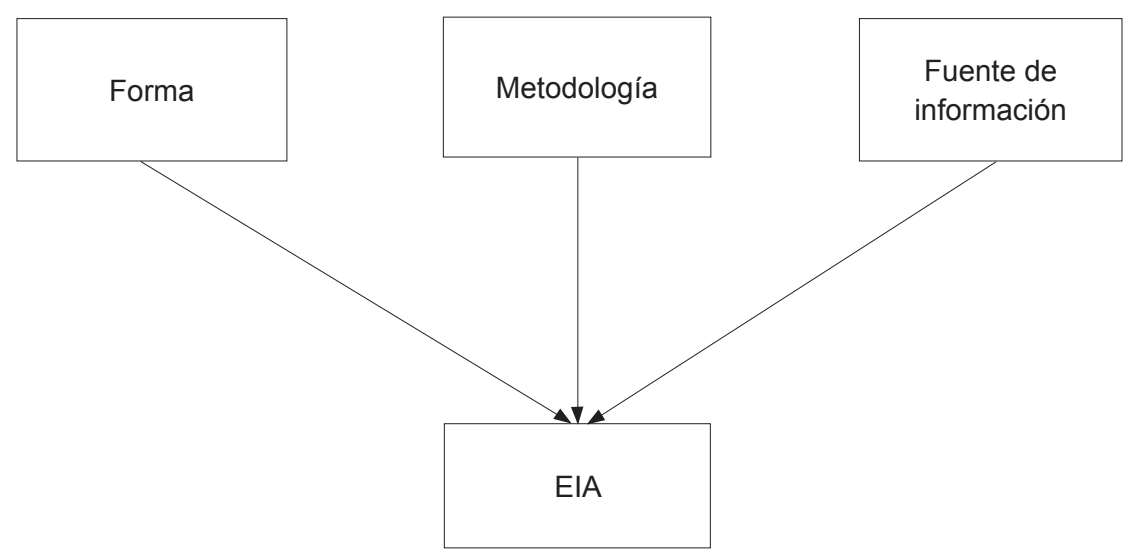

Figura 4: Posición crítica al estudio de impacto ambiental Fuente: Elaboración propia

La compensación y mitigación desde una perspectiva de la economía ecológica vinculada con la biología es el enfoque que prevalece en los Estudios de Impacto Ambiental (De Echave y Diez, 2013). A su vez, se pone en discusión las medidas compensatorias para reducir los impactos negativos de la actividad (Morán, 2012). No se cuestiona el enfoque para determinar el impacto, sino su veracidad. Por tanto, sostiene que no resulta clara la compensación (Proyecto justicia y responsabilidad corporativa, 2014).

El foco de la discusión ha sido el impacto sobre el flujo del agua y la compensación que garantiza su abastecimiento (Proyecto justicia y responsabilidad corporativa, 2014). Ello debido a que la empresa Yanacocha siempre se ha mostrado dispuesta a evaluar los mecanismos de compensación (La República, 2012).

La posición del Estado sobre la "devolución de la empresa a la comunidad" no está regulada en el sentido de cuantificar y determinar sus formas, y la empresa podía realizar una interpretación libre, como propone un representante del Ministerio del Ambiente (Ministerio del Ambiente, 2014). 
Al final, según sostiene un representante de la empresa Yanacocha, las empresas se "mueven" en base a leyes y estas son las condiciones en las que pueden realizar su actividad. La empresa en definitiva acepta "las reglas" y cuando se quieren cambiar "es donde vienen los problemas" (Méndez Lazarte, 2015). Por tanto, la empresa no propondría en sí un enfoque, sino que acepta el que propone el Estado.

La compensación ambiental se ha basado en la posibilidad de que Yanacocha construya cuatro reservorios de agua y que estos reemplacen las lagunas, proponiendo que sean construidos con tecnología de punta. Frente a esta alternativa, Marco Arana, uno de los líderes que más se ha opuesto al proyecto Conga, menciona: "Lamentablemente, el ser humano aún no ha aprendido a hacer lagunas" (Maquet, 2011).

En contraposición con este argumento, Roberto Parra indica que los reservorios son más beneficiosos que las lagunas, puesto que podrían asegurar el agua en época de sequía (Ponce, 2011).

Los estudios de impacto ambiental han estado orientados a la mitigación de los impactos y no a la compensación. En ese sentido, el estudio de impacto inicial no menciona a los reservorios como compensación; este surge posteriormente como una alternativa de solución al conflicto. Dicha compensación es colectiva, orientada a las poblaciones aledañas a la actividad extractiva y no es monetarizada, sino que se establecen comparaciones en la misma dimensión, es decir, metros cúbicos de agua perdida por el proyecto versus metros cúbicos de agua entregados en el reservorio.

En ese sentido, un funcionario del Ministerio del Ambiente cuestiona la monetarización con el argumento de que no se puede cuantificar ni predecir los impactos ambientales y menos aún monetarizarlos (Funcionario del Ministerio del Ambiente, 2014). Los reservorios de agua han sido la máxima compensación ofrecida por la probable falta de agua que el proyecto generaría (Radio Programas del Perú, 2013). Por ende, el debate se centra en la aceptación de la compensación propuesta (Lucar, 2011).

Las propuestas de compensación no están orientadas a la monetarización, pero tampoco recogen un enfoque basado en el ecosistema, sino más bien la compensación de un recurso por otro similar en igual o mayor dimensión, sin considerar los impactos que esto podría generar. Otra alternativa planteada es la realización de programas de desarrollo o sociales, que no corresponden a ningún enfoque de la economía.

Los pobladores, sin embargo, podrían mostrarse más cercanos a defender la compensación monetaria, como lo menciona Suarez (Suarez, 2014). Sin embargo, esta negociación es subjetiva y además el Estado indica que 
no puede intervenir por ser una negociación entre privados, generándose una disparidad entre las capacidades técnicas y de convencimiento de los pobladores de la comunidad y la empresa.

Finalmente, los ejercicios de valoración económica del proyecto han estado relacionados a la cuantificación de los ingresos económicos que generaría a los gobiernos regional y central, así como sus efectos en el empleo directo e indirecto (Albavera, 2015).

Por otro lado, en el estudio de impacto ambiental realizado por Knigth Piésold Consulting por encargo de la minera Yanacocha, y dentro de los seis impactos socioeconómicos generados, ubica el "incremento del valor de las tierras y propiedades no vendidas" como un impacto positivo alto (Radio Programas del Perú, 2011).

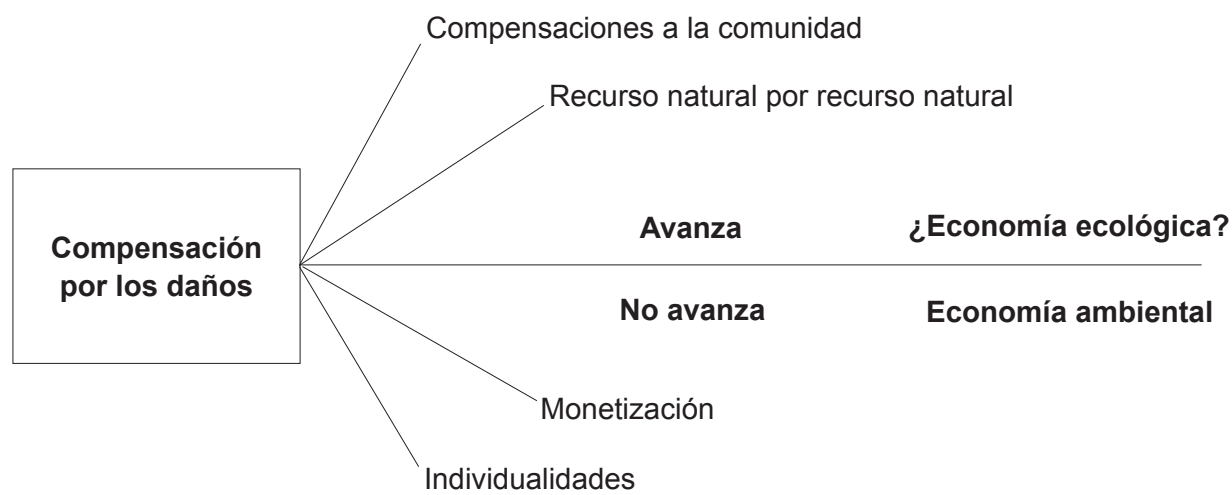

Figura 5: Posiciones con respecto a la compensación por el daño Fuente: Elaboración propia

Para representantes del Ministerio del Ambiente existe una valoración (Ministerio del Ambiente, Funcionaria del Ministerio del Ambiente, 2014), sin embargo, para el ex Ministro del Ambiente Ricardo Giesecke esta estaba subvaluada (Giesecke, 2011).

Otro de los elementos tomados en cuenta por los que buscan valorar el proyecto son los beneficios para los agricultores, considerando que son población directamente afectada, por ejemplo, Yanacocha (Actualidad Ambiental, 2012).

Los argumentos de valoración y su cuantificación no han sido debatidos y han tomado como única fuente los ingresos para el Estado, el 
empleo generado, la compensación al producir más agua y el incremento del valor de la tierra. Estas posturas han sido utilizadas mayoritariamente por los que apoyan el proyecto Conga y están más orientadas al enfoque de economía del bienestar y/o desarrollo económico.

\section{CONCLUSIONES}

La propiedad, uso y administración del recurso agua fue uno de los temas centrales en el caso Conga, y fue abordado desde una perspectiva conservacionista por parte de los líderes de la comunidades, que se centraron en argumentar la conservación natural de las lagunas y su entorno. Adicionalmente, se cuestionó el EIA, el documento más importante que resume los impactos ambientales del proyecto minero, argumentando que no considera una perspectiva holística, y en el último debate no aceptan los mecanismos de compensación por los daños, puesto que los nuevos reservorios artificiales no toman en cuenta la modificación del ecosistema. Todos estos argumentos encuentran un sustento en la economía ecológica.

En consecuencia, la investigación determina que existe una predominancia del enfoque de la economía ecológica en los argumentos de los actores que se oponen al proyecto minero Conga. El Estado peruano ha mencionado en múltiples oportunidades los beneficios de los proyectos mineros sin considerar los costos o riesgos ambientales, por ende, el enfoque de la economía ambiental no ha sido tabulado. Los funcionarios han mostrado argumentos diversos, algunos relacionados incluso con los enfoques de la economía ecológica y en breves momentos sobre la base de la economía ambiental.

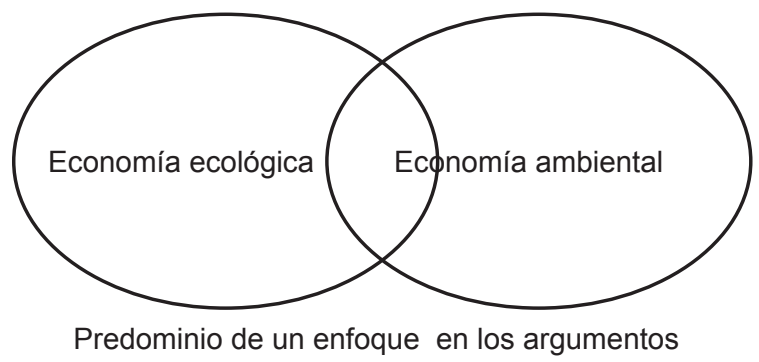

Figura 6: Enfoque que lidera el debate en el Perú Fuente: Elaboración propia 
En ese sentido, se puede afirmar que los conflictos ambientales se sustentan en las divergencias entre los enfoques económicos que asumen los líderes de las comunidades campesinas y los representantes del Estado, puesto que los primeros tienen un enfoque basado en la economía ecológica, mientras que las leyes del Estado y sus representantes no establecen con claridad una posición entre la economía ecológica y la ambiental.

En la medida en que el Estado peruano no defina con claridad una corriente de pensamiento desde la perspectiva de la economía positiva, le costará proponer políticas ambientales que consideren un enfoque económico. Sin embargo, existen indicios de que el gobierno, en el caso Conga, muestra un sesgo hacia el enfoque de la economía ambiental, sin ser asumido aún de manera preponderante, lo que en el Perú podría estudiarse en casos nuevos como Tía María y Las Bambas.

Las empresas mineras no asumen ninguno de los dos enfoques de la economía para explicar y analizar los problemas ambientales. Actúan en el marco del enfoque propuesto por el Estado.

El enfoque de la economía ecológica estuvo presente en el caso Conga como en otros casos en América Latina. Esta perspectiva motiva divergencias con la política ambiental actual, no solo del Perú, también en la región, motivando un debate que cuestiona los fundamentos de la política ambiental latinoamericana.

\section{REFERENCIAS BIBLIOGRÁFICAS}

Aburto, C. (2011). Edificador de la Economía Ambiental: homenaje a David W. Pearce. Gaceta de Economía, 16.

Actualidad Ambiental. (24 de febrero de 2012). Yanacocha lanza documental sobre los beneficios que traería el proyecto Conga en Cajamarca. Recuperado el 3 de diciembre de 2015 de Actualidad Ambiental: http:// www.actualidadambiental.pe/?p=14090

Aguilera Klink, F., \& Alcántara, V. (1994). De la economía ambiental a la economía ecológica. Barcelona: Icaria Fuhem.

Albavera, F. S. (2015). Conga en números. Caretas, 2241.

Arbeláez-Ruiz, Diana Carolina. (2014). La conflictividad en América Latina. Willaqniki.

Baker, S. E., y Edwards, R. (2012). How many qualitive interviews is enough? Expert voices and early carrer reflections on sampling 
and cases in qualitive research. National Centre of Research Methods Review Paper.

Bernales Perez, J. (12 de noviembre de 2014). Funcionaria del Ministerio del Ambiente. (C. Mendez Lazarte, Entrevistador)

Boyd, E. C. (Dirección). (2007). Tambogrande: Mangos, Murder, Mining [Película].

Centro Bartolomé de las Casas. (2010). Estudio de conflictos socioambientales en la subcuenta de Rio Santo Tomas-Provincia de Chumbivilcas, Región de Cusco. Lima.

Centro Nacional de Planeamiento Estratégico. (2010). Plan Perú 2021. Lima.

Coase, R. (1960). El Problema del Costo Social. The Journal of Law and Economics.

Congreso de la República del Perú. (1993). Constitución Politica del Perú de 1993.

Congreso de la Republica del Perú. (s.f.). Ley de Recursos Hidricos n. 29338.

Consejo Universitario UNC Universidad Nacional de Cajamarca. (mayo del 2012). Pronunciamiento. Recuperado en el 2014, de Universidad Nacional de Cajamarca: http://www.unc.edu.pe/noticias-y-eventos/ 4-pronunciamiento

Davis, R. y Franks. D. (2014). Costs of Company-Commynity Conflict in the Extractive Sector. Cambridge: Havard Kennedy School.

De Echave, J. D. (11 de noviembre de 2014). Ex Ministro del Ambiente. (C. Lazarte Méndez, Entrevistador)

De Echave, J., y Diez, A. (2013). Más allá de Conga. Lima: RedGE.

De Echave, J., Diez, A., Huber, L., Revesz, B., Ricard, X., y Tanaka, M. (2009). Minería y conflicto social. Lima: CBC, CIPCA, CIES, IEP.

Delacámara, G. (2008). Guía para decisores. Análisis económico de externalidades ambientales. Cepal.

El Comercio. (22 de octubre de 2014). Cajamarca: siguen las mentiras. El Comercio.

Ele, A. (16 de marzo de 2012). ¿En qué momento se jodió Conga? La Mula. 
Eyzaguirre, C. (5 de diciembre de 2014). Representante del Ministerio del Ambiente. (C. Mendez Lazarte, Entrevistador)

Flores, J. (11 de noviembre de 2014). Funcionario del Ministerio del Ambiente. (C. Méndez Lazarte, Entrevistador)

García, D., Kristrom, B., y Brannlund, R. (2005). Manual de Economía Ambiental y de los Recursos Naturales. Madrid: International Thomson Editores.

Georgescu-Roegen, N. (1970). La ley de la entropía y el proceso económico.

Gerencia de recursos naturales y gestión del medio ambiente Renama. (2011). Informe técnico sobre las Observaciones y Comentarios al Estudio de Impacto Ambiental del Proyecto Conga aprobado on Octubre de 2010. Cajamarca.

Giesecke, Ricardo. (25 de noviembre del 2011). Informe del Ministerio del Ambiente pide "mayor evaluación" del proyecto Conga. El Comercio.

Glave, M., y Kuramoto, J. (2007). La minería peruana: lo que sabemos y lo que aún nos falta por saber. Lima: Grade.

Gómez Orea, D. (2002). Evaluación de impacto ambiental: Un instrumento preventivo. Madrid: Mundi-Prensa.

Herrera Catalán, P., y Millones Destéfano, O. (2011). ¿Cuál es el costo de la contaminación ambiental minera sobre los recursos hídricos en el Perú? Lima: CIES, PUCP.

Huamaní, G. (5 de noviembre de 2014). Especialista en conflictos entre comunidades y empresas. (C. Mendez, Entrevistador)

Instituto de Ingenieros de minas del Perú. (2010). Minería peruana: Contribución al desarrollo económico y social. Lima: Área de Comunicaciones del IIMP.

Kapp, K. (1970). Social Costs, Economic Development, and Environmental Disruption. University Press of America.

Kapp, W. (1950). The social costs of business enterprise. Nottingham: Spokesman, Russell House.

Kemp, D., Owen, J., Cervantes, M., Arbeláez-Ruiz, D. C., y Benavides Rueda, J. (2013). Escuchando a la ciudad de Cajamarca. Queensland: CSRM, SMI. 
Knight Piésold Consultores S.A. (2009). Proyecto Toromocho Estudio de Impacto Ambiental. Lima.

La República. (26 de noviembre de 2011). Ministerio del Ambiente objeta el proyecto Conga. La República.

La República. (21 de abril de 2012). Yanacocha evaluará alternativas y mecanismos de compensación ambiental para Conga. La República.

La República. (6 de junio de 2013). Conga: Ronderos de Celendín advierten que darán sus vidas por lagunas. La República.

La República. (3 de febrero de 2015). Denuncian que Dinoes destruyó casa de campesina que gano juicio a Yanacocha. La República.

Lucar, N. (27 de noviembre de 2011). Conga. Lima.

Luke Brander, E. G.B.L. (2010). The economics of valuing ecosystem services and biodiversity. The Economics of Ecosystems and Biodiversity: The Ecological and Economic Foundations.

Malhorta, N. (2008). Investigación de mercado. México: Person Educación.

Maquet, P. (2011). Conga no va. Ideele. Lima.

Martinez Alier, J. (1998). Curso de Economía Ecologica. Organización de las Naciones Unidas.

Martinez Allier, J. (2010). El metabolismo social, los conflictos ambientales y los lenguajes de valoración. Encuentro Nacional de Economía Ecológica (p. 14). Bogotá: Universidad Nacional de Colombia .

Matthew, M. \&. (1994). Qualitative data analysis: an expanded sourcebook. Sage Production.

Méndez Lazarte, C. (2015). Conferencia sobre conflictos socioambientales. Transcripción del Conversatorio. Lima.

Mendezcarlo Silva, V., Medina Jiménez, A., y Becerra Quintero, G. E. (2010). Las teorías de Pigou y Coase, base para la propuesta de gestión e innovación de un impuesto ambiental en México. Recuperado el 2014, de Eumed. net: http://www.eumed.net/rev/tlatemoani/02/sjq.htm

Ministerio del Ambiente. (2011). Informe $n .{ }^{\circ}$ 001-2011. Lima.

Morán, R. (2012). El proyecto minero Conga, Perú: Comentarios al estudio de impacto ambiental (EIA) y temas relacionados. Colorado: EDLC. 
Naredo, J. M. (2011). Fundamentos de la economía ecológica. Madrid: CIPEcosocial.

Navero, J. (1987). La economía en evolución. Historia y perspectivas de las categorías básicas del pensamiento económico. Madrid: Siglo XXI.

Peru 21. (26 de agosto de 2012). Protestas contra Conga generaron pérdidas por más de 600 millones de soles. Peru 21.

Perú 21. (1 de marzo de 2013). Se reinician protestas contra Minas Conga. Perú 21.

Ponce, R. (15 de diciembre de 2011). A favor y en contra: El debate sobre el proyecto minero Conga. La Mula.

Proyecto justicia y responsabilidad corporativa. (2014). Expediente 36732013-AA. Toronto: Osgoode Hall Law School, York University.

Quispe, E. (1 de agosto de 2012). Noticias en Línea. Recuperado el 1 de diciembre de 2014, de Noticias en Línea: http://noticiasenlinea-efrain. blogspot.com/2012/08/el-78-de-cajamarquinos-rechaza-el.html

Radio Programas del Perú. (27 de diciembre de 2011). Mayor impacto económico local de Conga es incremento en valor de terrenos. Lima: Radio Programas del Perú.

Radio Programas del Perú. (10 de junio de 2013). Yanacocha: construcción de reservorios de Conga continuarán. Lima: Radio Programas del Perú.

Radio Programas del Perú. (10 de marzo de 2013). Yanacocha contruiría reservorios en Conga. Lima: Radio Programas del Perú.

Real Academía Española. (2014). Diccionario de la Real Academía Española.

Rivera-García, P. (s.f.). Marco Teórico. Elemento Fundamental en el Proceso de Investigación Científica. Zaragoza, España: UNAM.

Rodriguez, R. (2011). Informe n. ${ }^{\circ}$ 001-201-RRC/FHV Informe de evaluación Básica del Estudio de Impacto Ambiental (EIA)-Proyecto Conga Minera Yanacocha SRL.

Servicio Nacional de Certificación Ambiental. (2015). Senace. Recuperado el 5 de enero de 2015, de Senace: http://www.senace.gob.pe/nosotros/ que-es-el-senace/ 
Suarez Rojas, L. A. (2014). Avatares por el agua. Ruralidad, mega-infraestructuras y desigualdades persistentes en el Perú. Lima: Programa Cybertesis Perú.

Suarez, L. (7 de noviembre de 2014). Especialista de conflictos basados en agua. (C. Mendez Lazarte, Entrevistador).

Torres, E. (26 de noviembre de 2014). (C. Mendez, Entrevistador).

Wagner, L. S. (2010). Problemas ambientales y confllicto social en Argentina. Movimientos socioambientales en Mendoza. La defensa del agua y el rechazo a la megaminería en los inicios del siglo XXI. Argentina.

Walter, M. (2008). Nuevos conflictos ambientales mineros en Argentina. El caso Esquel (2002-2003). Revista Iberoamericana de Economía Ecológica, 8, 15-28.

Xercavins Valls, J., Sabater Pruna, A., Cervantes Torre-Marín, G., y Cayuela Marín, D. (2005). Desarrollo Sostenible. Barcelona: UPC Universitat Politécnica de Ctalunya.

Yin, R. K. (1984). Case Study Research. Design and Methods. London: Sage Publications. 